\title{
Villes en crise ? Les politiques municipales face aux pathologies urbaines (fin $\mathrm{XVIII}^{\mathrm{e}}$-fin $\mathrm{XX}^{\mathrm{e}}$ siècle)
}

\section{Michel Biard}

\section{(2) OpenEdition \\ Journals}

Édition électronique

URL : https://journals.openedition.org/ahrf/1959

DOI : 10.4000/ahrf.1959

ISSN : $1952-403 X$

Éditeur :

Armand Colin, Société des études robespierristes

Édition imprimée

Date de publication : 1 décembre 2005

Pagination : 251-253

ISSN : 0003-4436

Référence électronique

Michel Biard, "Villes en crise? Les politiques municipales face aux pathologies urbaines (fin xVIII-fin xx $x^{\mathrm{e}}$ siècle) ", Annales historiques de la Révolution française [En ligne], 342 I octobre-décembre 2005, mis en ligne le 05 avril 2006, consulté le 22 avril 2022. URL : http://journals.openedition.org/ahrf/1959 ; DOI : https://doi.org/10.4000/ahrf.1959

Ce document a été généré automatiquement le 22 avril 2022

Tous droits réservés 


\title{
Villes en crise ? Les politiques municipales face aux pathologies urbaines (fin XVIII ${ }^{\mathrm{e}}$-fin $\mathrm{XX}^{\mathrm{e}}$ siècle)
}

\author{
Michel Biard
}

\section{RÉFÉRENCE}

Yannick MAREC (dir.), Villes en crise ? Les politiques municipales face aux pathologies urbaines (fin XVIII'-fin XX siècle), Paris, Créaphis, 2005, 768 p., ISBN 2-913610-49-8, $32 €$

1 Ce gros volume rassemble les actes du très important colloque qui a réuni à Rouen, en décembre 2002, une cinquantaine d'intervenants autour du thème de la croissance urbaine, de ses maux (réels ou fantasmés) et des politiques municipales mises en œuvre pour y faire face. Cinq parties rythment l'ensemble: les désordres urbains et leurs régulations; les réponses apportées; pauvreté, précarité et politiques d'assistance; expériences de politiques sociales urbaines aux $\mathrm{XIX}^{\mathrm{e}}$ et $\mathrm{Xx}^{\mathrm{e}}$ siècles ; autour des politiques de la ville. Le lecteur est ainsi entraîné sur la piste des premiers fantasmes sécuritaires que font naître les " classes dangereuses ", des questions liées aux logements insalubres et à la pauvreté, des tentatives pour gérer au mieux des villes de plus en plus peuplées et étendues, le tout en passant naturellement par les grandes cités françaises, mais aussi par "Vienne-la-Rouge ", Cordoue, Turin ou encore Bruxelles (pour ne citer que quelques-unes des nombreuses villes ici abordées).

2 L'immense majorité des communications a porté sur les $\mathrm{XIX}^{\mathrm{e}}$ et $\mathrm{XX}^{\mathrm{e}}$ siècles, seules quatre d'entre elles se rattachent au XVIII ${ }^{e}$ siècle et/ou à la Révolution française, et doivent donc être mentionnées dans les A.H.R.F. C'est tout d'abord le cas d'un texte de Vincent Milliot sur l'action du lieutenant général de police de Paris, Lenoir, entre 1775 et 1785. Grâce à diverses archives et aux Mémoires de Lenoir, l'auteur nous livre la vision d'une " police " urbaine bien difficile à mettre en œuvre dans le "monstre " démographique parisien, que ce soit dans ses aspects de contrôle social, d'organisation de l'espace 
public (par exemple avec le développement des marchés), d'amélioration de la salubrité (notamment avec l'éloignement des cimetières vers la périphérie), sans oublier bien sûr les classiques nécessités du maintien de l'«ordre ». Par son constant souci de tout coordonner, mais aussi d'inciter ses commissaires à doubler la répression des infractions d'une sorte de pédagogie visant à éduquer les habitants, Lenoir esquisse ce que Vincent Milliot nomme une "police des Lumières", conçue « comme un véritable laboratoire des moyens de lutte contre les pathologies urbaines, dont certains sont promis à se développer au siècle suivant ».

Deux autres communications portent également sur une partie de ces préoccupations de la police urbaine (au sens du mot entendu au XVIII ${ }^{e}$ siècle, que vient d'étudier Paolo Napoli dans son ouvrage consacré, en 2003, à La naissance de la police moderne). Dietlind Hüchtker analyse les "stratégies et tactiques" de la police des subsistances à Berlin, entre 1770 et 1850 , ce qui le conduit avant tout à étudier le rôle des femmes dans l'espace urbain. Dans le cadre de ce qu'il nomme «Bitt- und Bettelkultur» (culture de la sollicitation et de la quête), l'auteur évoque notamment deux activités qui leur permettent de survivre, tout en étant strictement réglementées ou interdites: la mendicité et la prostitution. Difficile apparemment de sévir contre mendiants et mendiantes de Berlin, car souvent une partie de la population, voire même les policiers et soldats chargés de la répression, prennent leur défense...! Il est vrai que, d'une part, l'aumône est présentée comme une vertu chrétienne et que, d'autre part, gagner son «misérable petit bout de pain " peut être défini comme une des diverses manières de gagner sa subsistance et non comme de la mendicité. Quant à la prostitution, les ambiguïtés sont évidemment là encore de mise, puisque l'interdiction rigoureuse par la police laisse toute leur place aux bordels enregistrés par cette même police. Le règlement de 1792 insiste sur le fait que cette activité qui heurte la morale chrétienne doit se faire le plus discrètement possible, ainsi les maisons de plaisirs illicites doiventelles être fermées côté rue et bien entendu écartées des rues les plus animées. Quelques prostituées, chassées d'une salle de danse par un fonctionnaire de police, s'empressent de ramener les débats moraux chers aux élites sociales à quelques réalités tristement élémentaires: "Par cette interdiction trop sévère, nous sommes et serons malheureusement complètement ruinées, car elle nous a ôté notre liberté, par laquelle nous gagnons notre pain » (souligné par moi). C'est aussi de la misère et des tentatives pour la " prendre en charge » que traite la communication d'Armelle El Kaïm, dans le cadre des villes de Haute-Normandie pendant la période révolutionnaire. Après avoir rappelé qu'il s'agit là d'un souci ancien, à travers le travail des intendants, celui de l'Assemblée provinciale créée en 1787, et bien sûr l'activité des administrations du département, des districts et des communes organisés à partir de 1790 (avec, par exemple, l'établissement des bureaux de bienfaisance en 1791, treize à Rouen plus un bureau central), l'auteur insiste, à juste titre, sur la politique esquissée en l'an II avec la loi du 22 floréal qui ouvre le "Grand Livre de la Bienfaisance nationale ». Aussitôt, en effet, les administrations de Seine-Inférieure relaient le rapport présenté par Barère le 22, comme à Rouen où la municipalité en donne lecture trois jours plus tard. Les communes reçoivent les demandes d'inscription qui sont alors transmises aux districts, puis au département qui établit une liste des indigents à secourir. Dans ce département, pour le premier semestre de l'an III, cent quarante-sept citoyens figurent sur cette liste et se partagent 8180 livres apportées par l'État. L'aide est nationale, la mise en œuvre est locale, mais le tout ne survit pas à la fin de la Convention dite «thermidorienne ». Comme l'écrit l'auteur, « cette bienfaisance montagnarde fut de courte durée ». Si l'on 
pourrait sans doute nuancer quelque peu l'emploi de cet adjectif « montagnard», force est de constater la pérennité du paupérisme dans les villes ici étudiées.

Reste la quatrième et dernière communication, celle de Raymonde Monnier, sous le titre «L'impératif patriotique de la Commune de Paris sous la Révolution ». C'est cette fois de pratiques politiques qu'il s'agit, avec avant tout l'exemple de la Commune issue de l'insurrection du 10 août 1792, dans laquelle les militants cordeliers jouent un rôle décisif. L'auteur rappelle la pratique des pétitions collectives, chère au cœur des militants sectionnaires, et développe les cas précis de trois pétitions collectives qui ont circulé dans les sections de Paris au printemps 1793 lors de la crise finale conduisant à la perte de pouvoir des «Brissotins » (28 mars, 15 avril et 30 mai). Des cartes (p. 541) viennent utilement compléter la démonstration. Enfin, sont évoquées les fêtes qui ont eu lieu en 1793 et 1794 dans l'espace parisien en hommage aux martyrs de la Liberté, culte relancé ici par le passage de la vague déchristianisatrice et qui est lié aux impulsions données par les Cordeliers, les sociétés populaires, mais aussi la Commune elle-même (celle-ci, par ses Affiches, dispose notamment d'un bon support pour donner davantage de publicité aux initiatives diverses venues des militants populaires). L'auteur conclut en soulignant bien sûr le paradoxe de cette Commune qui tente d'élaborer une "synthèse patriotique " en encourageant les pratiques militantes, et aide « à l'instauration du gouvernement révolutionnaire et de la Terreur, qui devaient précipiter sa chute et la mise au pas du mouvement populaire».

Plusieurs autres communications intéresseront aussi les lecteurs des A.H.R.F., notamment celles qui portent sur les toutes premières années du XIXe siècle (ainsi celle de Pierre Guillaume sur le coût des pathologies urbaines et leur prise en charge à Bordeaux, ou encore celle d'Alexandre Nugues-Bourchat sur le quadrillage policier à Lyon, ville vue de la capitale comme l'une des plus dangereuses pour le pouvoir central, notamment depuis 1793). Plusieurs des rapports introductifs sont par ailleurs dus à des auteurs bien connus pour leurs travaux sur la période révolutionnaire (Jean-Pierre Jessenne, Guy Lemarchand, Pascal Dupuy). Enfin, cela va de soi, ceux qui liront les actes de ce colloque ne pourront qu'être passionnés, en tant que citoyens aussi bien qu'en tant qu'historiens, par des problématiques qui sont toujours d'une actualité brûlante dans nos villes à l'aube d'un xxI siècle où hélas nombre des maux urbains subsistent. Comment à cet égard ne pas souligner une fois de plus que le mot « cité », mot chargé de sens s'il en est, désigne désormais pour beaucoup de nos concitoyens les quartiers des périphéries urbaines qui concentrent la plupart des problèmes sociaux? Les mises en garde de Jean-Claude Vimont et Jean-Claude Farcy, qui ouvrent les actes du colloque sur le thème de "Louis Chevalier "revisité" ", seraient sans doute une saine lecture à conseiller à ceux qui ne pensent qu'à "nettoyer " des "cités " perçues comme criminogènes. Nul doute que ce colloque suscitera des travaux nouveaux dans un futur proche, mais l'idéal, dont il est toujours permis de rêver un peu, serait qu'en sus les auteurs aient pu donner à penser aux responsables politiques d'aujourd'hui. 